\title{
MEDIA PSYCHOLOGYY SU APROXIMACIÓN A LA PSICOFISIOLOGÍA: UNA DISCIPLINA PARA ANALIZAR LOS PROCESOS MEDIÁTICOS
}

Media Psychology and it's Approximation to Psychophysiology: A Field of Study to Analize Media Processes

Media Psychology e a sua aproximação à psicofisiologia: uma disciplina para analisar os processos mediáticos

Rodero, Emma. Universidad Pompeu Fabra (España)
emma.rodero@upf.edu

Larrea, Olatz. Universidad Pompeu Fabra (España)

olatz.larrea@upf.edu

Mas, Lluís. Universidad Pompeu Fabra (España)

lluis.mas@upf.edu

Fecha de recibido: 14 de octubre de 2015

Fechas de aprobado: 14 de abril de 2016

\section{RESUMEN}

Esta propuesta tiene como objetivo presentar Media Psychology como una disciplina en los estudios de comunicación que busca analizar y entender la respuesta o reacción de los individuos cuando se exponen a mensajes 
mediáticos. Así pues, el objetivo principal de este artículo es explicar la concepción y evolución de esta disciplina y, especialmente, cómo se puede aplicar a la comunicación. En particular, este análisis se centrará en el paradigma psicofisiológico que puede enmarcarse dentro de esta disciplina. Este paradigma aplicado a la comunicación se centra en estudiar el procesamiento cognitivo y emocional de los individuos por medio de la medición de las respuestas psicofisiológicas del cuerpo durante su exposición a los mensajes mediáticos: ritmo cardíaco, conducción de la piel, movimiento muscular o dilatación de las pupilas, entre otros parámetros. Los datos obtenidos por medio de estos instrumentos se pueden complementar con diferentes escalas, técnicas cualitativas o mediciones de comportamiento que permiten ofrecer una visión más completa de la experiencia de los individuos expuestos a mensajes mediáticos.

Palabras clave: Media Psychology, psicofisiología, procesamiento de información, procesos mediáticos.

\section{ABSTRACT}

This paper aims at presenting Media Psychology as a discipline in communication studies to analyze and understand the individuals' response when are exposed to media messages. Therefore, the main objective of this paper is to explain the origin, the development and especially the application of this discipline to communication. In particular, this analysis will focus on the psychophysiological paradigm that can be framed within this discipline. This paradigm applied to communication studies the cognitive and emotional processing by measuring the psychophysiological responses of the body during media messages exposure: heart rate, skin conductance, facial EMG or expansion of pupils, among other parameters. The data obtained by using these instruments can be complemented with different scales, techniques or qualitative measures of behavior, which can offer a more complete perspective of the individuals' experience exposed to media messages.

Keywords: Media Psychology, psychophysiology, processing of information, mediated processes.

\section{RESUMO}

Esta proposta tem como objetivo apresentar Media Psychology como uma disciplina nos estudos de comunicação que busca analisar e entender a resposta ou reação dos indivíduos quando se expõem a mensagens mediáticos. Portanto, o objetivo principal deste artigo é explicar a concepção, a evolução e especialmente como se pode aplicar esta disciplina à comunicação. Em particular, esta análise se entrará no paradigma psicofisiológico que pode enquadrar-se dentro desta disciplina. Este paradigma aplicado à comunicação se centra em estudar o processamento cognitivo e emocional dos indivíduos através da medição das respostas psicofisiológicas do corpo durante a sua exposição às mensagens mediáticas: ritmo cardíaco, condução da pele, movimento muscular ou dilatação das pupilas, entre outros parâmetros. Os dados obtidos através destes instrumentos se podem complementar 
com diferentes escalas, técnicas qualitativas ou medições de comportamento que permitem oferecer uma visão mais completa da experiência dos indivíduos expostos a mensagens mediáticas.

Palavras-chave: Media Psychology, psicofisiología, processamento de informação, processos mediáticos.

\section{Introducción}

¿Qué le ocurre a nuestro cuerpo cuando contemplamos la imagen de una apetitosa hamburguesa en una pantalla de ordenador? Seguramente al verla nuestra boca va a comenzar a salivar. Un beso en una serie de televisión puede hacer que nuestro corazón se acelere. 0 el sonido de una acción precipitada en un partido de fútbol en la radio puede provocar que nuestras manos suden. Cada vez el ser humano está más expuesto a diferentes estímulos que recibe de diferentes medios: los medios de comunicación, Internet, redes sociales. Imágenes, sonidos, olores, formas y movimientos recibidos por medio de todo tipo de mensajes mediáticos en un entorno cada vez más digital y complejo. El problema es que la capacidad de procesamiento es limitada. Eso significa que no se puede procesar al mismo tiempo mucha información. Por eso, se está frente a la obligación de seleccionar solo aquellos estímulos que son relevantes. El cerebro debe filtrar de entre todos los estímulos que recibe aquellos que son importantes para dar sentido y sobrevivir en el mundo. La pregunta entonces que cabe hacerse es, ¿cómo lo hace?, ¿cómo reacciona el cerebro, cómo responde y procesa esos mensajes mediáticos? Para responder a esta pregunta, en los años ochenta nació la disciplina Media Psychology. La idea se basaba en comprender cuál era la influencia de la tecnología en el comportamiento humano. Es ahí donde la psicología y la comunicación convergen, dando lugar después al actual paradigma de estudio científico. Con el paso del tiempo, dentro de esta disciplina han surgido teorías provenientes de la comunicación que tratan de explicar cómo se produce esta interacción dinámica entre los sujetos y los mensajes de los medios de comunicación. Una de las teorías más influyentes es el modelo de capacidad limitada de la información mediática formulado por Lang (2009). Esta teoría toma de la psicología formulaciones teóricas y la metodología psicofisiológica y las aplica a los procesos dinámicos de comunicación producidos por los medios de comunicación. Se trata, por tanto, de una concepción y aproximación novedosa al análisis de los efectos que los medios producen y que puede contribuir a superar la actual crisis en que se encuentra la disciplina de la comunicación. Así pues, el objetivo de este artículo es mostrar las posibilidades que tiene esta disciplina utilizando como ejemplo el paradigma psicofisiológico. En primer lugar, se mostrará el proceso de evolución que ha dado lugar a esta disciplina; en segundo lugar, se explicará el paradigma; y, en tercer lugar, se analiza cómo Media Psychology puede ayudar a superar la crisis de la comunicación. 


\section{Historia de Media Psychology: ¿de dónde venimos?}

Desde su concepción a mediados del siglo xx, el estudio de la comunicación de masas ha evolucionado como resultado de los cambios en el contexto sociocultural y de la revolución tecnológica, aun en pleno desarrollo (Dicken-Garcia \& Viswanath, 2002; McQuail, 2013; Shome, 2006). Diferentes teorías y modelos afloraron en el marco del llamado paradigma de los efectos que ha sido probablemente la tradición con mayor influencia en los últimos cincuenta años (Neuman \& Guggenheim, 2011).

Sin embargo, los estudios de comunicación considerados genuinos (Mass Communication Research) nacen, precisamente, cuando los científicos de otras disciplinas (sociología con Paul F. Lazarfeld, ciencia política con Harold Lasswell y psicología social con Carl Hovland y Kurt Lewin) cuestionan la gran teoría de los efectos. El papel de la radio durante la Segunda Guerra Mundial provocó la aparición de dos importantes trabajos: The Psychology of Radio (1935), de Cantril y Allport, y The Invasion from Mars (1940), de Cantril, Gaudet y Herzog, que analizaron los efectos de la famosa emisión radiofónica La guerra de los mundos. Paralelamente, autores como Schramm (1954) consideraron las partes del proceso comunicativo como unidades de análisis: emisor, receptor, mensaje, canal y contexto.

El final de la Segunda Guerra Mundial trajo consigo un nuevo paradigma científico que surgió de las ciencias físicas. El empirismo radical derivó en el empirismo construccionista, en el que el relativismo y la teoría sistémica dominaban sobre la experimentación pura y la medición. Del mismo modo, en los estudios de comunicación, el campo respondió con dos aproximaciones opuestas: el funcionalismo de Pearson (1978) y la teoría crítica (Horkheimer, 1937). El funcionalismo adoptó la tradición positivista y se concentró en la consolidación de los métodos de las ciencias sociales con la finalidad de acumular evidencias en la teoría de los efectos de los medios. Al mismo tiempo, Shannon y Weaver, que trabajaban para la compañía Bell's Phone, formularon la Teoría Matemática de la Información como una vía para medir el canal, lo que, junto con los principios cibernéticos de Turing, se convirtió en la base de la incipiente revolución tecnológica. La eficacia de la comunicación se concebía como la diferencia entre el mensaje enviado y el recibido, mientras que la entropía se entendía como la cantidad de información nueva codificada y descodificada. Aunque la teoría matemática nunca consideró los aspectos semánticos de este código, la comunicación fue concebida desde ese momento como un mensaje en proceso de producción y de recepción. Esta concepción es clave para entender el paradigma de los efectos de los medios, pues la comunicación es un sistema donde el mensaje tiene el propósito de reducir la incertidumbre mediante un conjunto de creencias, actitudes, valores y situaciones sociales compartidas entre emisor y receptor. Como principio constitutivo, la teoría de la información postulaba que la comunicación es factual y empírica. Además, con la aparición de la semiótica, el estructuralismo y la lingüística moderna, el análisis del mensaje fue más sistemático, aunque ninguna de estas perspectivas buscó correlacionarlo con los efectos en la recepción.

En 1967, el biólogo austriaco Ludwig von Bertalanffy formalizó la Teoría General de Sistemas (1973) como procedimiento científico que incorporaba los principios de la teoría de la información y la cibernética en el nuevo paradigma de la ciencia. Esta teoría intentaba integrar disciplinas nuevas de las ciencias sociales en el marco general de la ciencia. Esta teoría afirmaba que ningún sistema natural puede existir completamente aislado, sino que debe interaccionar con factores externos para sobrevivir. Además, las entidades que formaban parte de ese sistema interaccionaban entre ellas para cumplir una función de orden superior que a su vez servía a la función del sistema. 
Durante esta década, diferentes teorías se han derivado del estudio social de los efectos: la agenda setting, la espiral del silencio y la teoría del cultivo.

Estas teorías se articularon a partir de los primeros axiomas puros sobre los efectos de los medios. Sin embargo, los resultados muestran hipótesis unidimensionales sobre la correlación entre la estructura textual de los mensajes y las actitudes, sentimientos o comportamientos de los receptores. En la actualidad, estas teorías han continuado acumulando evidencias y han incrementado la complejidad de sus supuestos mediante el agrupamiento contextual y social de los receptores. Con todo, el poder predictivo de estas teorías es aún pobre, pues los efectos de los medios se consideran lineales, fragmentados y limitados (Demers, 2000; Lang, 2013). Los problemas de la tradición de los efectos de los medios con la consideración de un efecto poderoso de los medios hizo surgir otras teorías, como la teoría del cultivo (cultivation theory o cultivation analysis) de Gerbner y Gross (1976), centrada en estudiar la manera en que los individuos viven en la sociedad a través de la mediatización de los medios de comunicación, especialmente la televisión. La otra teoría crítica con la concepción tradicional de los efectos de los medios fue la teoría de los usos y gratificaciones, preocupada por los motivos por los que los individuos consumen mensajes de los medios de comunicación. La aproximación sistémica a la comunicación intenta superar estos problemas bajo la consideración de este campo como un proceso de interacción dinámica.

En este contexto, Media Psychology nace como una disciplina para abordar los cambios provocados por el uso de las nuevas tecnologías. Surge, por tanto, fruto del cambio de paradigma en la psicología: de la psicología del comportamiento - behaviorism - al procesamiento de la información (Lachman, Lachman \& Butterfield, 1979; Miller, 2003; Potter, 2013). Este cambio de perspectiva dio lugar al estudio de los procesos mentales producidos por la exposición a los mensajes mediáticos. Se trata de un intento por analizar qué ocurre en la mente, denominada por los behavioristas hasta entonces black box, ya que hasta el momento era concebida como una caja negra que no podía estudiarse. Media Psychology surge, por tanto, de un campo de estudio científicamente establecido, la psicología, y aplica el conocimiento de esta disciplina a la forma en que el ser humano se relaciona con la tecnología e interacciona con los mensajes de los medios de comunicación. Conscientes de la creciente influencia de la tecnología, la American Psychological Association fundó en 1986 la división 46, dedicada a su estudio. La idea era comprender cuál era la influencia de la tecnología en el comportamiento humano. Media Psychology emplea la perspectiva de la psicología para entender la compleja relación entre los seres humanos y el entorno mediático. De esta manera, los orígenes de esta disciplina se producen en el campo de la psicología y en su interacción con la tecnología. Sin embargo, en los años posteriores esta concepción se considera de aplicación también desde los estudios de comunicación. Se entiende que es en esta interacción ser humano-tecnología donde la psicología y la comunicación pueden converger. Así es como nace un nuevo paradigma de estudio científico también desde la disciplina de la comunicación. En este sentido, según Giles (2003) aparecen dos diferentes aproximaciones al estudio del procesamiento de los mensajes: la psicofisiología, como método para entender y estudiar la black box desde la comunicación (Lang, 2000); y la llamada revolución cognitiva desde la psicología (ver los trabajos de Reeves, 1986).

Desde entonces, la investigación en el marco de este campo ha ido evolucionando mediante la creación de una revista con el mismo nombre, Media Psychology, indexada en comunicación, o de programas académicos, especialmente de Máster, sobre esta disciplina emergente. En 2003 se puso en marcha el primer programa de doctorado con ese mismo nombre en la Fielding Graduate University en Estados Unidos. Junto con esto, en las 
facultades de comunicación se comenzaron a crear algunos laboratorios dedicados a estudiar el procesamiento cognitivo de los mensajes mediáticos, primero en Estados Unidos y últimamente en Europa, especialmente en Alemania. Por una parte, las investigaciones han permitido formular axiomas sofisticados sobre el sistema cognitivo humano. Por otra parte, se ha adoptado gran parte del conocimiento acumulado sobre la estructura, el contenido y la función de los medios y los mensajes. La evolución ha sido tal que en 2011 la búsqueda del término en Google produjo más de 100000 resultados entre artículos, páginas web y otros recursos (Brown, 2013). Por tanto, si bien los comienzos se originan en la psicología con aplicación en algunos aspectos de la comunicación, hoy en día la disciplina se trabaja tanto en psicología como en comunicación.

Existen muchas definiciones de Media Psychology, todas ellas complementarias. En su origen, Media Psychology, desde la psicología, sirve para intentar dar una explicación general a cómo los procesos mediados por la tecnología impactan en el ser humano. En otras palabras, Media Psychology analiza cómo el cerebro interacciona con un entorno mediatizado por la tecnología. En la actualidad, y desde la perspectiva de la comunicación, Media Psychology se concibe en sentido más amplio y no centrado exclusivamente en la tecnología, sino en el ser humano como procesador de mensajes. Por tanto, es el estudio científico acerca de cómo los individuos perciben, procesan y son potencialmente influenciados por contenidos significativos de un mensaje mediatizado. Existen también una gran variedad de definiciones de mensaje mediatizado. De hecho, algunas personas pueden pensar que la comunicación cara a cara es comunicación mediatizada por el aire y la luz. Sin embargo, la mayoría de investigadores se centran en algún tipo de mediación electrónica, sea cinemática o televisiva, musical o radiofónica, etc. Es importante destacar que la disciplina ha superado ya la restrictiva concepción de los medios entendidos como los tradicionales (prensa, radio, televisión...) para extender sus análisis a todo tipo de comunicación mediatizada (videojuegos, Internet, redes sociales...).

Dentro de la disciplina Media Psychology existe una variedad de temas y ámbitos que pueden estudiarse mientras impliquen el análisis de cualquier tipo de interacción dinámica comunicativa entre un sujeto y un estímulo. Un estudio realizado por Baker Derwin y De Merode (2013) examinó los temas más tratados en la revista Media Psychology entre 1999 y 2010. En los últimos años, los estudios tienen que ver especialmente con la ética, publicidad, marketing, arte, noticias, actitud, género, identidad, política, educación, narrativa y salud. Así pues, se puede observar que las aplicaciones pueden ser variadas tanto en el ámbito (salud, género, política), o en el género (publicidad, noticias) como en el análisis del comportamiento, la cognición o las emociones. Por número de menciones, el medio televisivo fue el más citado, aunque empieza a extenderse el análisis a los videojuegos y las redes sociales. El mayor número de contribuciones en número de artículos a la revista lo registró la investigadora estadounidense Annie Lang.

Como disciplina, Media Psychology representa un paradigma que integra diversas concepciones en un intento de explicar las complejas interacciones comunicativas que hoy en día mantienen los individuos con los medios, sean del tipo que sean. En este campo, existen diversas aproximaciones teóricas dentro de la disciplina. Algunas de las más importantes son la teoría del procesamiento de la información social (social information processing theory) de Crick y Dodge (1994); la teoría del aprendizaje social (social learning theory) de Montgomery (1996); la teoría cognitiva social (social cognitive theory) de Bandura (1973, 1991); el modelo general de agresión (general aggression model) de Bushman y Anderson (2001); y la teoría del procesamiento limitado de información mediática (Lang, 2009). 
La teoría del procesamiento de la información social se centra en el estudio del procesamiento cognitivo y emocional que se produce en una interacción social, por tanto, en cómo los individuos se relacionan en la sociedad. En segundo lugar, la teoría del aprendizaje social estudia el comportamiento de los individuos como modo de conocer cómo aprenden en un entorno social. En los últimos años, se está aplicando a cómo se aprende de los nuevos medios, especialmente de Internet y de las redes sociales. Muy relacionada con esta se encuentra la teoría cognitivo-social de Bandura, una figura clave para explicar el paso del conductismo al cognitivismo. Esta teoría también se centra en el análisis de la conducta por medio de la observación para conocer cómo se produce el aprendizaje social. Por su parte, el modelo general de agresión analiza la agresión y la violencia desde una perspectiva cognitivo-social. Por último, la teoría del procesamiento limitado de información mediática es una de las más influyentes en la disciplina. Esta teoría se llamó después Limited Capacity Model Motivated Mediated of Message Processing (LC4MP) y representa una aproximación teórica al modelo de procesamiento cognitivo de la información, centrándose en estudiar la atención y la memoria de los receptores expuestos a mensajes de los medios de comunicación (Lang, 2006, 2009). Además, proporciona un conjunto de instrumentos de medición psicofisiológica que permiten al investigador registrar la respuesta biológica de los sujetos expuestos a los mensajes y, con ello, realizar un análisis de la interacción producida entre un mensaje, el medio de comunicación y el sujeto receptor. En definitiva, se trata de un modelo motivacional basado en el análisis de las respuestas psicofisiológicas de los individuos ante los estímulos mediáticos (Lang, 2009; Lang, Bolls \& Potter, 2009). Por tanto, todos estos modelos tienen en común que se centran en el análisis del procesamiento cognitivo-emocional de los individuos para explicar distintos comportamientos y reacciones, generalmente derivados de la interacción de los medios de comunicación. Por supuesto, no son las únicas teorías que han surgido en el campo, pero estas ilustran de manera clara el objetivo central de la disciplina Media Psychology.

Debido a la variedad de teorías, en Media Psychology se emplean también una gran variedad de métodos tanto cuantitativos como cualitativos. Sin embargo, los cualitativos (análisis de contenido, estudios etnográficos...) son minoritarios en la disciplina. La mayoría de los estudios emplean métodos experimentales cuantitativos diversos debido a su origen en el behaviorismo. En este sentido, se toma de la psicología el método experimental. Generalmente, primero se identifica una interacción comunicativa (por ejemplo, cómo los anuncios de televisión sobre tabaco pueden influir en que los fumadores abandonen el hábito), entonces, se establecen las hipótesis y, por último, se mide esta interacción por medio de diversos métodos, generalmente con el esquema pre-test/pos-test. La forma más común de análisis ha sido de varianza (ANOvA). En este sentido, se puede hablar de dos grandes métodos a la hora de analizar la interacción mediática: el análisis del comportamiento y el análisis psicofisiológico. El análisis del comportamiento es central en algunas teorías, como la teoría cognitiva social, y se centra en el estudio de la conducta de los individuos mediante la observación. En este sentido, una de las teorías más influyentes es el paradigma de la exposición selectiva (Selective Exposure Paradigm), que postula que los individuos buscan contenidos mediáticos que satisfagan sus necesidades cognitivas y emocionales (Knobloch-Westerwick, 2014). El otro método es el llamado paradigma psicofisiológico o Media Psychophysiology (Bartholow \& Bolls, 2013). Este paradigma es central al modelo de capacidad limitada de procesamiento de la información mediática (LC4MP) formulado por Lang (2009) desde los estudios de comunicación. Por supuesto, estos métodos se pueden complementar con escalas, encuestas, tests y con los métodos cualitativos (análisis de contenido, encuestas, entrevistas, 
grupos de discusión...). Este texto se centrará en el paradigma psicofisiológico, por tanto, en el apartado siguiente se explican las principales variables metodológicas.

\section{Media Psychophysiology: utilización de medidas psicofisiológicas en comunicación}

Dentro de la disciplina Media Psychology ha surgido el paradigma Media Psychophysiology que específicamente aplica medidas psicofisiológicas al estudio y análisis del procesamiento de la información mediática por parte de los receptores (Bartholow \& Bolls, 2013; Potter \& Bolls, 2012). La utilización de medidas psicofisiológicas no es nueva en modo alguno. La psicofisiología es una disciplina consolidada en el campo de la psicología y está a caballo entre esta y la fisiología. Surge en los años cincuenta en Estados Unidos y se materializa en la creación de la Sociedad para la Investigación Psicofisiológica en 1960 y en la revista científica Psychophysiology en 1964 (Vila \& Guerra, 2009). Lo que resulta novedoso aquí es aplicar los métodos empleados por esta disciplina para analizar procesos comunicativos y, en especial, los mensajes procedentes de los medios de comunicación. Su principal ventaja es que permite registrar la respuesta del individuo en tiempo real durante la exposición al mensaje mediático. Así pues, no se le pregunta posteriormente si ha prestado o no atención al mensaje o qué impacto emocional ha tenido, lo cual implica confiar en la respuesta del individuo, sino que se registra su reacción fisiológica en el mismo momento. Esta reacción permite conocer el impacto cognitivo y emocional que el mensaje ha producido. Se sabe que los individuos tienen a veces problemas para identificar sus reacciones, pensamientos o emociones, o dificultades para transmitirlos o comunicarlos, o simplemente no quieren hacerlo o escogen respuestas convenientes. Esta metodología no presenta estos problemas y, como puede complementarse con otras técnicas (por ejemplo escalas o encuestas a posteriori), se convierte en un método que aporta información más fiable.

La psicofisiología busca dar sentido a la relación entre lo que se piensa, se siente y se hace con aquello que el cuerpo refleja fisiológicamente. Por tanto, se basa en entender los procesos psicológicos a partir de la medición de la respuesta fisiológica del cuerpo. Para conocer cómo se interpreta la respuesta psicofisiológica, es preciso encontrar el correlato o indicador del proceso psicológico correspondiente a los diferentes cambios fisiológicos. Para ello, se precisa un conocimiento básico del funcionamiento del sistema nervioso humano.

El sistema nervioso central se ramifica por todo el cuerpo por medio del sistema nervioso periférico. Puesto que la interacción física con el entorno se produce mediante el cuerpo (mirando, tocando, oliendo, con movimiento...), la medición de las respuestas del sistema nervioso periférico durante la interacción comunicativa es relevante para conocer el procesamiento que un individuo realiza de un mensaje.

El sistema nervioso periférico es el conjunto de nervios que parten del sistema nervioso central hacia todas las zonas del cuerpo y realizan funciones sensitivas y motoras. Se divide en el sistema nervioso autónomo y en el sistema nervioso somático-sensorial. El sistema nervioso autónomo está formado por los nervios aferentes y eferentes de los órganos internos, es independiente del control voluntario y puede dividirse en el sistema nervioso simpático y parasimpático. El sistema nervioso simpático está formado por los nervios procedentes de la parte torácica y lumbar de la medula espinal. Es el que activa el cuerpo de forma automática como resultado de una situación de amenaza inmediata y lo predispone para la acción/actividad. Produce la respuesta lucha-huida. Por 
su parte, el sistema nervioso parasimpático está formado por los nervios procedentes de la zona cervical. Este sistema coordina la actividad en reposo en procesos como, por ejemplo, la digestión o el sueño. Por tanto, tiene una función de conservación de la energía. Por otro lado, el sistema nervioso somático está formado por fibras aferentes y eferentes de los órganos sensoriales y motores. Y su función consiste en enviar información sensorial al sistema central y controlar la actividad muscular.

Dependiendo de qué sistema se analice, existen tres técnicas de análisis psicofisiológico: las que miden el sistema nervioso central, el sistema nervioso somático o el sistema nervioso autónomo (Vila \& Guerra, 2009). Entre las relacionadas con el sistema nervioso central se encuentran la electroencefalografía (EEG), los potenciales evocados (ERPS) o la imagen por resonancia magnética funcional (fMRI), que miden la actividad eléctrica del cerebro y que ya han comenzado a utilizarse en estudios de comunicación. Entre las técnicas que miden el sistema nervioso somático se encuentran la electromiografía, la actividad respiratoria, los movimientos oculares o la actividad laríngea. Por último, entre las que registran el sistema nervioso autónomo se encuentran la actividad eléctrica de la piel o conductancia, la actividad cardiovascular, la actividad salivar, el movimiento de las pupilas, la actividad gastrointestinal y la actividad sexual (Vila \& Guerra, 2009). De ellas, las más empleadas en este paradigma aplicado a los medios de comunicación han sido principalmente el ritmo cardíaco, la conductancia de la piel y el movimiento muscular facial por medio de la electromiografía.

En primer lugar, el pulso o tasa cardíaca es la principal medida psicofisiológica de la actividad cardiovascular. Se registra mediante un electrocardiograma (EKG) que mide los latidos por minuto. Operativamente, el pulso cardíaco se mide colocando tres electrodos en los brazos del participante (Potter \& Bolls, 2012). La deceleración del ritmo cardíaco se relaciona con el reflejo de orientación, es decir, con una respuesta orientada y un incremento en la atención. Esta respuesta orientada representa una respuesta simpática fásica, es decir, ocurre en un momento específico durante la exposición a una información novedosa o relevante. Esta respuesta orienta la atención hacia el mensaje y mide el esfuerzo cognitivo realizado por el individuo. Una deceleración del ritmo cardíaco en un periodo de entre 5 y 7 segundos es indicadora de un aumento en el esfuerzo cognitivo y en la atención (Potter \& Bolls, 2012). Sin embargo, esta disminución del ritmo cardíaco también se ha asociado con la valencia negativa de los mensajes (Lang, 1995).

En segundo lugar, dentro de este modelo, la electromiografía facial se emplea para medir la dimensión de valencia de un mensaje, aunque también puede emplearse para medir el sobresalto o el grado de tensión o distensión de un sujeto. La dimensión de valencia se refiere a la respuesta positiva o negativa a la información en procesamiento. En ausencia de un nivel alto de activación, el sistema simpático se activa al exponerse a contenido positivo. Sin embargo, si el mensaje produce un elevado nivel de activación, el sistema aversivo se activa más rápido en los mensajes negativos que el sistema simpático ante mensajes positivos. Por tanto, "it is negative messages that receive more intense-processing at a comparatively lower level of arousing content than positive messages" (Lang et al., 2009, p. 199). Mediante la electromiografía facial se registra la actividad eléctrica procedente de la contracción de las fibras musculares (Vila \& Guerra, 2009), midiendo la activación de los músculos faciales; los más comunes son el músculo cigomático, el orbicular y el corrugador. El primero está situado en la parte superior de la mejilla. Su activación es indicadora de una respuesta emocional positiva. Por ejemplo, si alguien finge una sonrisa, su boca podría indicar la mueca de una sonrisa, pero faltaría el músculo cigomático para ser fiable. Los dos últimos se sitúan en los ojos. El orbicular rodea el ojo y es indicador de una emoción positiva. El corrugador 
se encuentra situado sobre la nariz entre las cejas y su activación indica una respuesta emocional negativa, como cuando se frunce el ceño. Para medir la actividad de estos músculos, se deben emplazar dos electrodos en cada uno de ellos.

En tercer lugar, la tercera técnica psicofisiológica más empleada en este modelo y también en la psicología en general es la medición de la actividad electrodérmica (EDA). También es llamada conductancia de la piel o respuesta galvánica de la piel, entre otros nombres. Esta medición se basa en registrar la actividad de las glándulas ecrinas que se encuentran principalmente en la palma de la mano. Por eso, en este caso, los electrodos se colocan en la superficie de la palma de la mano del participante, aunque también puede medirse en la planta del pie, si el sujeto tiene las manos ocupadas (manipulando un videojuego, por ejemplo). Su correlato psicológico es el nivel de activación o arousal representada por la intensidad de activación emocional provocada por un estímulo. Por tanto, esta variable se utiliza para medir el nivel de activación de la respuesta, así como el esfuerzo cognitivo y atención ante mensajes con diferentes niveles de complejidad. Cuanto mayor sea la amplitud de respuesta, mayor grado de activación, reacción emocional y atención hacia el estímulo.

Las respuestas psicofisiológicas se pueden producir de forma continua en el tiempo durante la exposición a un estímulo, por ejemplo, mientras se escuchan las noticias en la radio - respuesta tónica- o bien en un momento específico del estímulo, por ejemplo, en el momento en que un individuo ve la imagen de un hombre asesinado en las noticias en televisión. En este caso, se parte de un nivel de referencia (baseline) que es alterado en ese momento preciso produciendo una respuesta fásica (Potter \& Bolls, 2012).

En este sentido, el equipo de Annie Lang en Indiana University ha realizado durante los últimos veinte años un gran trabajo de investigación sobre las variables psicológicamente relevantes en los mensajes mediáticos y su respuesta cognitiva y emocional. Sus estudios se han centrado en analizar la respuesta a elementos estructurales tanto en televisión (cambios y movimientos de cámara...), en radio (cambios de tipo de voz, música, efectos de sonido...) y en la web (animación, imágenes emocionales...) utilizando metodología psicofisiológica. Así pues, estos trabajos han demostrado la validez de este método para analizar cómo los individuos procesan los mensajes procedentes de los medios de comunicación.

A continuación, para explicar cómo esta metodología se puede aplicar en los estudios de comunicación, se presenta un estudio más en profundidad que analiza las variables prosódicas de la voz en la publicidad radiofónica y otros dos estudios más a modo de ejemplo para ilustrar otras variables.

El primer estudio muestra la aplicación de esta teoría al análisis del procesamiento del lenguaje mediante las variaciones de los elementos prosódicos (Rodero \& Potter, 2015). El objetivo general de este proyecto era determinar si diferentes estrategias prosódicas de entonación aplicadas a mensajes publicitarios de radio podían mejorar la atención y la memoria de los oyentes con el fin de facilitar su procesamiento cognitivo. Para ello, se diseñó un experimento intra-sujetos en el que 50 participantes escucharon 12 diferentes comerciales que contenían 3 modelos de entonación. Los dos primeros modelos mantenían un mismo nivel de tono - grave o agudo-sin variaciones durante todo el comercial. El otro modelo tenía variaciones internas de tono comenzando desde un tono agudo en la primera parte de la oración y acabando en grave en la segunda parte. La metodología empleada se basó en escalas auto-perceptivas acerca de la efectividad y la adecuación de las variaciones tonales aplicadas a los comerciales de radio, las mediciones psicofisiológicas del ritmo cardíaco, la conductancia de la piel y el movimiento de los músculos faciales (orbicular y corrugador) y, por último, tests de medición del recuerdo y del reconocimiento. 
Por tanto, se emplearon un conjunto de métodos cuantitativos para analizar la respuesta de los participantes durante y después de la exposición a los diferentes espacios publicitarios radiofónicos. En este artículo, y a modo de ejemplo, solo se explicarán los datos obtenidos en las mediciones psicofisiológicas.

La asignación de recursos cognitivos y la atención fue medida mediante la frecuencia cardíaca (EKG). Este indicador fue registrado en pulsaciones por minuto (BPM) (Potter \& Bolls, 2012). Como ya se ha explicado, varios estudios aplicados al procesamiento de los mensajes mediáticos han demostrado que una desaceleración del ritmo cardíaco es indicativo de una respuesta de orientación y, por consiguiente, señala una mayor atención (Graham \& Clifton, 1966; Potter \& Bolls, 2012). Para medir el ritmo cardíaco, durante la presentación de los estímulos se emplearon dos electrodos Beckman $\mathrm{Ag}-\mathrm{AgCl}$ de $8 \mathrm{~mm}$ que se colocaron en los antebrazos de los participantes con el electrodo de tierra en el antebrazo no dominante.

La valencia emocional de los anuncios publicitarios de radio fue analizada mediante la electromiografía facial (Facial EMG). Para ello, se midió la actividad de dos músculos faciales: el corrugador para la emoción negativa y el orbicular para la positiva usando dos pares de electrodos Beckman mini-Ag-AgCl. Dos electrodos se colocaron debajo del ojo en la parte exterior (músculo orbicular) y los otros dos electrodos fueron colocados en la superficie de la piel a lo largo de la ceja (en el músculo corrugador). Por último, el grado de activación (arousal) fue medido mediante la actividad electrodérmica (EDA), que detecta cambios en las propiedades de conductancia de la piel. La actividad electrodérmica se cuantificó mediante dos electrodos $\mathrm{Ag}-\mathrm{AgCl}$ de $8 \mathrm{~mm}$ colocados en la palma de la mano no dominante del sujeto.

Puesto que se trata tan solo de un ejemplo, no se entrará a fondo en los resultados, pero los siguientes gráficos pueden dar una idea del tipo de análisis que puede realizarse. En relación con el ritmo cardíaco, los resultados mostraron que los sujetos atendieron más al nivel agudo-grave. Este modelo de entonación tuvo la media más baja (93) seguido del tono grave (94) y el tono agudo (96).

Figura 1. Ritmo cardíaco

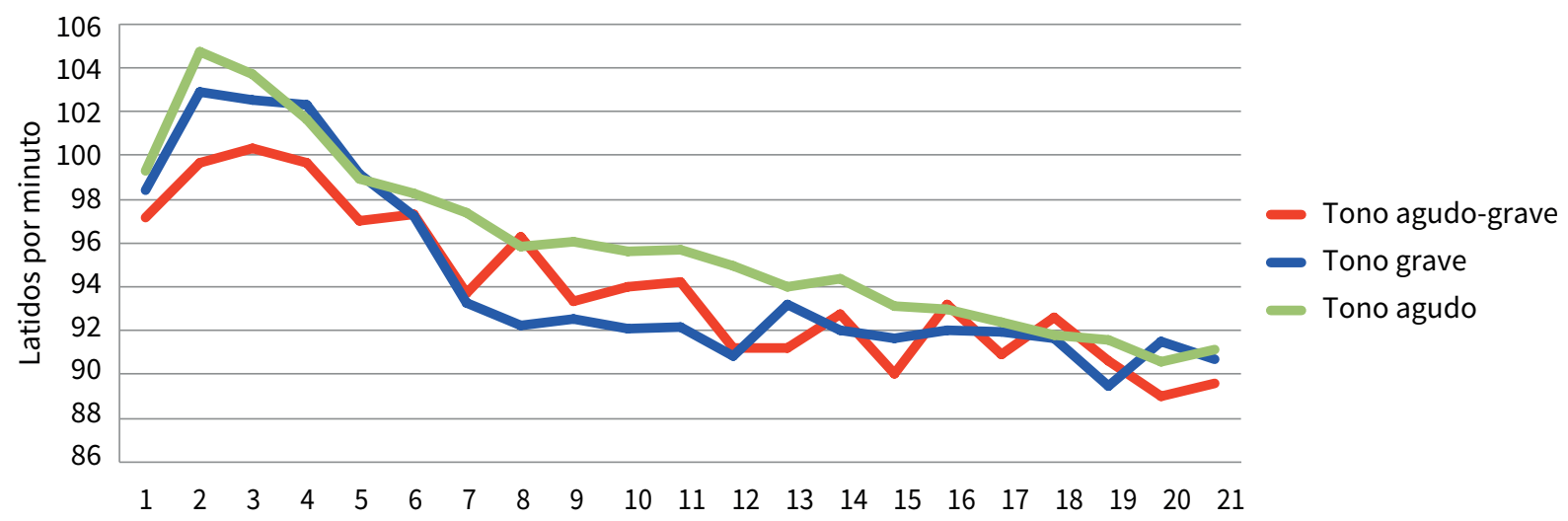

Fuente: elaboración propia. 
En relación con el movimiento de los músculos, los resultados fueron más dispersos, aunque en líneas generales puede observarse que el movimiento del músculo orbicular mostró que la emoción más positiva la consiguió el modelo agudo-grave, con una media más elevada, como muestra la figura 2.

\section{Figura 2. Movimiento del orbicular}

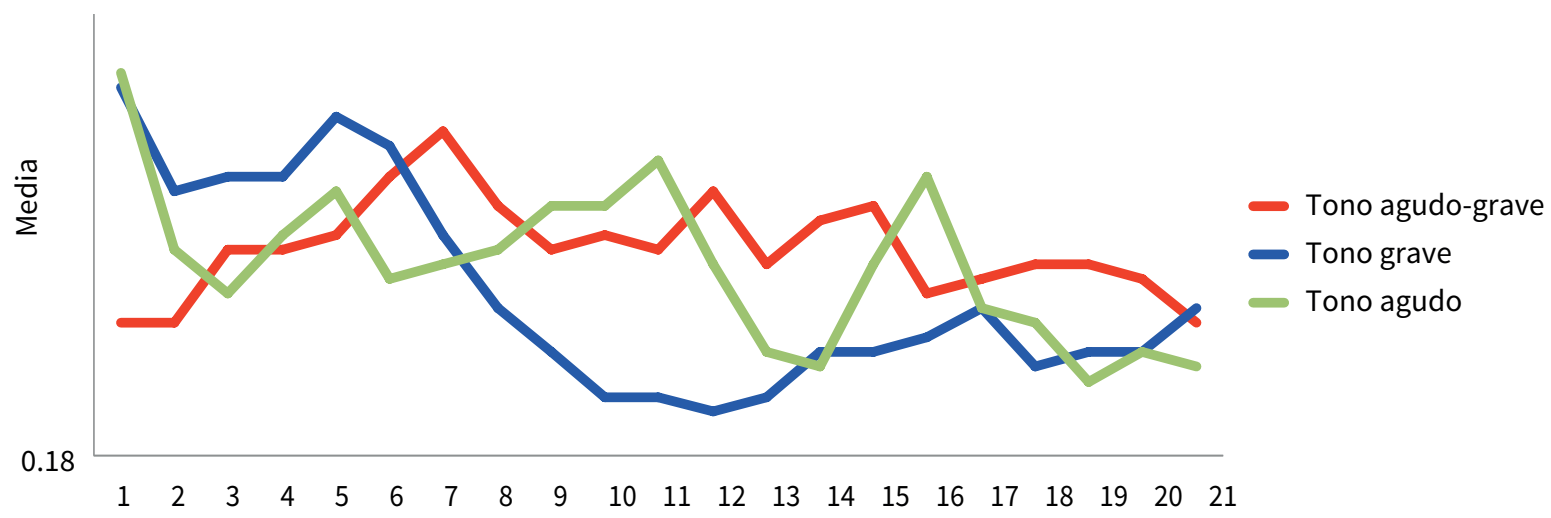

Fuente: elaboración propia.

Segundo, el músculo corrugador mostró unos resultados más claros donde la emoción más negativa la suscitó principalmente el nivel grave seguido del agudo, como se observa en la figura 3.

Figura 3. Movimiento del corrugador

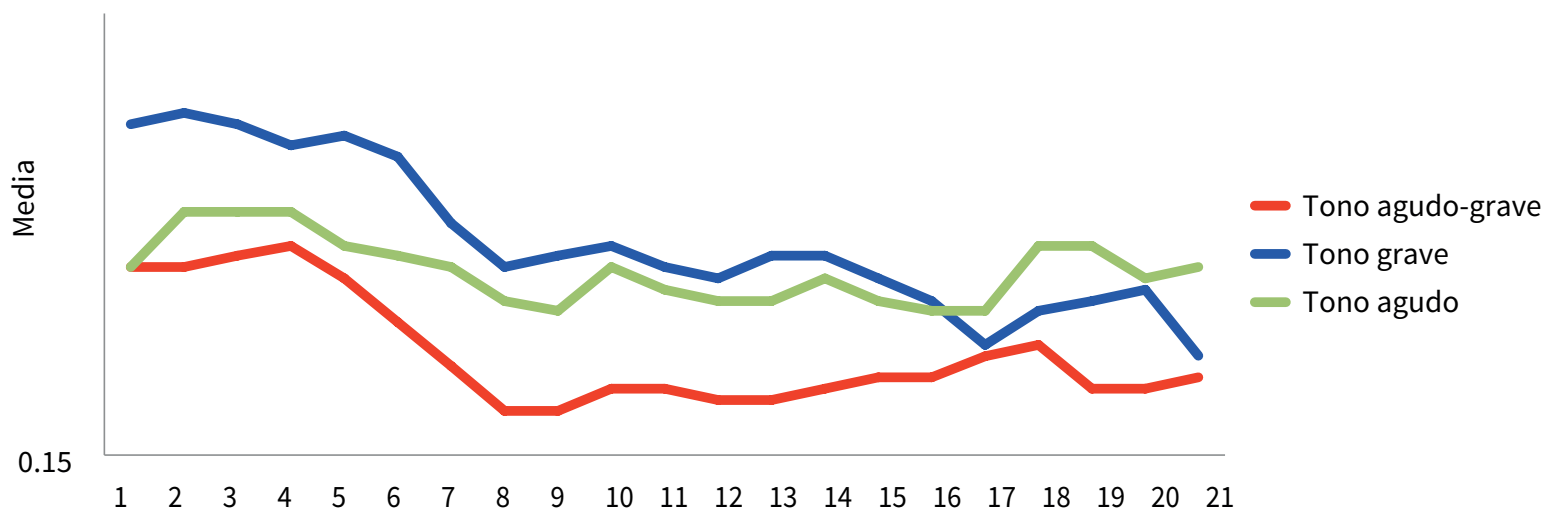

Fuente: elaboración propia.

Por último, la actividad electrodérmica indicó que el nivel agudo-grave fue con diferencia el que más nivel de activación logró conseguir, como se refleja claramente en la figura 4. 
Figura 4. Actividad electrodérmica

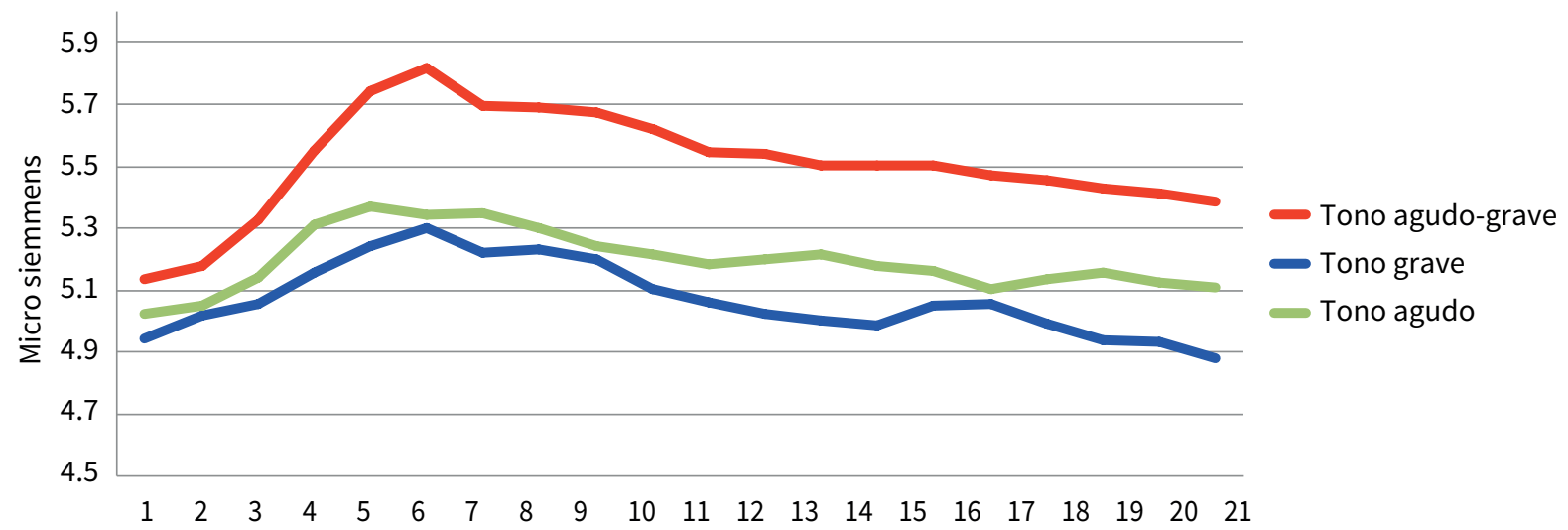

Fuente: elaboración propia.

En conclusión, y sin entrar aquí en detalle, el nivel agudo-grave es el que mostró un mejor comportamiento, puesto que fue el que más esfuerzo cognitivo y nivel de activación provocó junto a una emoción positiva sostenida y una emoción negativa baja.

Como este estudio, otras investigaciones han sido aplicadas a distintas variables comunicativas analizadas utilizando metodología psicofisiológica. A modo de ejemplo, Leshner, Bolls y Thomas (2009) publicaron un estudio para comprobar qué tipo de mensajes eran más efectivos en la publicidad antitabaco: aquellos cuyo contenido explicaba las amenazas sobre la salud del consumo de tabaco, calificados como mensajes de miedo, y aquellos que intentaban generar aversión mediante imágenes gráficas que mostraban las consecuencias del consumo de tabaco sobre los diferentes órganos, calificadas como mensajes de aversión. El objetivo del estudio era saber qué nivel de miedo o aversión facilitaba un mejor procesamiento del mensaje. Al tratarse de mensajes con un alto componente emocional, era probable que se activara el sistema motivacional aversivo, con lo que la respuesta orientada del sujeto aumentaría y el nivel de reconocimiento de la información empeoraría. Esto se ha denominado la cascada defensiva (Bradley, Codispoti, Cuthbert, \& Lang, 2001). Pero lo importante aquí es que para medir el grado de atención, la respuesta orientada y la asignación de recursos cognitivos al estímulo se utilizó el ritmo cardíaco. Como se ha explicado anteriormente, una disminución del ritmo cardíaco puede indicar una respuesta orientada del sujeto y un incremento de la atención. La variación del ritmo cardíaco fue calculada cada segundo respecto del punto de referencia inicial. En este caso, los resultados indicaron que los mensajes con miedo o aversión altos provocaron una mayor deceleración del ritmo cardíaco y un mejor reconocimiento de la información que los de bajo miedo o aversión. Sin entrar en más detalles, estos resultados confirmaron la gran capacidad que tienen los mensajes altamente emocionales para generar respuestas orientadas, así como la existencia de la cascada defensiva. Por lo tanto, los datos obtenidos, medidos aquí por medio del ritmo cardíaco, ayudaron a caracterizar la respuesta atencional de los sujetos ante estos mensajes y a ofrecer información para diseñar con más precisión este tipo campañas publicitarias. 
El segundo de los estudios que a modo de ejemplo se explicó aquí se centra en medir las emociones y las reacciones faciales a un estímulo. Weyers, Mühlberger, Hefele y Pauli (2006) se propusieron investigar las reacciones musculares faciales de un grupo de participantes ante las expresiones faciales de avatares estáticos (neutro, feliz, enojado) y dinámicos (morfo desarrollo de neutral a felices o enojados). El estímulo fue presentado durante un segundo cada uno y las respuestas fueron medidas a través de electromiografía facial (EMG). Los registros indicaron reacciones de emoción específica a los avatares felices, que se reflejaron en el aumento del movimiento del músculo cigomático mayor y en la disminución de la tensión en el corrugador, con reacciones más importantes a las expresiones dinámicas en comparación con las estáticas. A pesar de estar clasificadas como más intensas, las expresiones de enojo no provocaron ninguna activación significativa en el corrugador. Con estos datos, los autores llegaron a la conclusión de que las reacciones faciales a las expresiones de enojo y felicidad cumplen diferentes funciones en las interacciones sociales.

Estos son solo algunos ejemplos de las posibilidades de aplicar la psicofisiología a la comunicación. De la misma manera que ocurre con estas variables, cualquier proceso comunicativo puede ser analizado mediante esta metodología, la cual aporta datos psicológicamente relevantes.

\section{Media Psychology y su aportación a la comunicación}

Algunos de los principales autores creen que Media Psychology es algo más que una metodología, una teoría o incluso una sub-disciplina de la comunicación. De hecho, Media Psychology presenta en la actualidad todo lo que un paradigma científico requiere, como es la definición acotada de un fenómeno de estudio y diferentes formas específicas de medirlo. Esta disciplina también formula preguntas de investigación consensuadas, recoge conjuntos de datos que se apoyan sobre constructos teóricos validados y presenta una comunidad de académicos que estudian cómo la gente percibe, procesa y potencialmente es influenciada por los medios.

Puesto que muchos investigadores coinciden en que la comunicación es un campo en crisis (Lang, 2013), esta disciplina podría ser una manera de superar los problemas con que actualmente se encuentra. Por un lado, existe una gran fragmentación de teorías que estudian de forma solapada diferentes objetos de estudio. Además, no hay forma de saber qué teoría explica mejor un determinado fenómeno porque todas ellas utilizan aproximaciones y métodos diferentes y abordan diferentes objetos de estudio (Craig, 1999, 2007). La diversidad entre las secciones de estudio de las seis principales organizaciones internacionales en comunicación es solo un ejemplo de la mezcla de tradiciones que actualmente integra la disciplina (Mas \& Rodero, 2015). Asimismo, los efectos estadísticos atribuidos a los medios hasta el momento son bastante limitados (Lang, 2013). Por tanto, parece poco probable que las actuales líneas puedan conseguir modelos cerrados sobre los efectos de los medios. En cambio, la aproximación contenida en Media Psychology implica concepciones epistemológicas, teóricas y metodológicas coherentes entre sí. El modelo evolucionado del procesamiento limitado de la información mediática, Dynamic Human-Centered Communication Systems Theory o DHCCST (Lang, 2014) reclama la superación de las tradicionales correlaciones lineales con los efectos mediáticos y defiende un modelo multimodal de estados cognitivos cualitativos. Estos estados deben acabar por definir todos los factores relevantes para el proceso comunicativo (Bartholow \& Bolls, 2013).

En primer lugar, epistemológicamente, este modelo toma la comunicación como un sistema dinámico de estados cognitivos entre dos o más cerebros en interacción que buscan sobrevivir. Si un cerebro interacciona con 
otros, diremos que es comunicación. De acuerdo con esta concepción de la comunicación, los humanos no pueden parar de interaccionar. Incluso cuando estamos dormidos, los sentidos continúan activos, sobre todo el oído, pero también la visión por medio de los sueños. No se puede evitar interaccionar, responder o reaccionar corporalmente a los motivos del entorno queimpactan psicológica y emocionalmente. En este contexto, los medios son parte del entorno con el que constantemente se interacciona. El objetivo entonces es estudiar los elementos de los mensajes que son psicológicamente relevantes para mantener la interacción comunicativa. Estos elementos pueden ser formales o de contenido. De esta forma, el sistema comunicativo implica dos subsistemas complejos (el mensaje y el ser humano) interaccionando de forma dinámica y recursiva.

En segundo lugar, este paradigma tiene una clara implicación teórica: por primera vez, la psicología y los psicólogos provenientes del paradigma de procesamiento de la información dan importancia al estudio del mensaje. En otras palabras, este mensaje no es un simple estímulo, sino un complejo sistema comunicativo en sí mismo. Por lo tanto, la naturaleza dinámica y compleja de los mensajes no es solo un problema metodológico sino una cuestión teórica que debe ser abordada teniendo en cuenta el conocimiento acumulado durante tantos años de investigación sobre los efectos de los tipos de mensajes y contenidos.

Y, en tercer lugar, este paradigma implica una perspectiva metodológica más integral proveniente de otra disciplina, la psicofisiológica, que, junto con otros métodos de recogida de datos, ofrece una aproximación holística al análisis del proceso comunicativo. Esto significa que este paradigma debe considerar las tres fuentes de datos informativos sobre la experiencia humana: el canal fisiológico, a partir del cual se obtienen medidas psicofisiológicas, el canal auto-perceptivo, que implica preguntar a las personas sobre sus experiencias, y el canal conductual u observacional, que conlleva técnicas de observación participante y no participante. La medición de la conducta incluye la observación del comportamiento del participante antes, durante y después de la exposición a los medios. Las medidas autoperceptivas vienen representadas por escalas de medida validadas (Likert, Osgood...), así como por auto-informes (grupos de discusión, entrevistas en profundidad...) en los que cada participante evalúa su reacción al mensaje. Y las medidas psicofisiológicas registran la respuesta fisiológica en tiempo real (el pulso, la conductancia de la piel y la electromiografía facial, entre otras). Estas tres fuentes de datos pueden ser clasificadas en cuatro dimensiones (Martínez, 2012) - sentir, pensar, decir y hacer-. Por lo tanto, este paradigma debe incluir el análisis de las reacciones emocionales (sentimientos), de la cognición humana (pensamiento) y de sus actitudes y comportamientos (expresiones y acciones) por medio de medidas psicofisiológicas de los estados emocionales y cognitivos, (encuestas, entrevistas, escalas...) para captar la expresión explícita de los individuos y técnicas de observación de su comportamiento y actitudes. En suma, los métodos cuantitativos y cualitativos son totalmente complementarios. Aunque, como se ha comentado antes, esta idea no es nueva (Lang, 1979), sí es la primera vez que los principales investigadores intentan incorporarla de forma generalizada a los modelos comunicativos. La integración de estas tres fuentes de datos parece uno de los caminos más apropiados para avanzar metodológicamente en las ciencias de la comunicación.

\section{Conclusiones}

La disciplina Media Psychology, aunque se heredera del paradigma clásico de los efectos, introduce un enfoque de estudio que puede dar una posible respuesta a la actual crisis de los estudios en comunicación. En la actualidad, 
no hay consenso en torno a una definición de comunicación y de su objeto de estudio. Las diferentes líneas de investigación de las principales revistas científicas, los temas de las conferencias o las secciones de las organizaciones para el estudio de la comunicación aglutinan diferentes criterios solapados y desordenados: industriales, formales, de contenido o al servicio de la moda de investigación imperante. En caso contrario, ¿cómo sería posible tener diferentes teorías sobre comunicación y salud, radio, sonido, persuasión o comunicación y mujeres sin que se produzca apenas interacción alguna entre ellas?

En este sentido, Media Psychology puede contribuir a superar la actual crisis de la comunicación cambiando la manera en que hasta ahora se ha concebido y analizado el proceso comunicativo. La base teórica general que se deriva es la conexión empírica entre un mensaje y unos efectos sobre los receptores. Primero, considera que la comunicación es un proceso dinámico de interacción entre variables psicológicamente relevantes de un mensaje y un sistema humano. Segundo, la formación de las variables dependientes es precisa y completa: los postulados y los constructos teóricos tienen un correlato directo con medidas exactas del objeto. Y tercero, su aproximación holística - con las tres fuentes de información sobre la experiencia humana y los estados emocionales y sociales humanos, especialmente la psicofisiología- puede ayudar a mejorar el rigor de los estudios de comunicación.

Sin duda alguna, aún queda mucho trabajo por hacer. De hecho, la definición acotada de las variables del mensaje no se ha producido. Las investigaciones realizadas han tratado variables formales (como el tamaño de la imagen o sus dimensiones, los colores o formas, la longitud de los planos, etc.), variables de contenido (emoción, narración, ritmo, publicidad de diferentes temáticas, etc.) y variables mediáticas (audio, imagen, dispositivos personales, videojuegos, etc.). Pero no ha habido una formalización integral de estas como elementos relevantes de un sistema que interacciona en el seno de un modelo con los diferentes estados del sistema humano.

En conclusión, Media Psychology, y dentro de ella el paradigma psicofisiológico, planteada como una disciplina holística, integral y conciliadora con los avances hasta ahora realizados, parece una alternativa ilusionante para el estudio científico de la comunicación.

\section{Referencias}

1. Baker, D. E., \& De Merode, J. (2013). Inside Media Psychology: The Story of an Emerging Discipline as Told by a Leading Journal. In E. Baker Derwin (Ed.), The Oxford Handbook of Media Psychology (pp. 75-95). Oxford: Oxford University Press.

2. Bandura, A. (1973). Aggression: A social learning analysis. Englewood Cliffs, NJ: Prentice-Hall.

3. Bandura, A. (1991). Social cognitive theory of self-regulation. Organizational behavior and human decision processes, 50(2), 248-287.

4. Bartholow, B. D., \& Bolls, P. (2013). Media Psychophysiology: The brain and beyond, In K. Dill (ed.), The Oxford Handbook of Media Psychology (474-495). New York: Oxford University Press.

5. Bolls, P. D., Lang, A., \& Potter, R. F. (2001). The Effects of Message Valence and Listener Arousal on Attention, Memory, and Facial Muscular Responses to Radio Advertisements. Communication Research, 28(5), 627-651.

6. Bradley, M. M., \& Lang, P. J. (2000). Affective reactions to acoustic stimuli. Psychophysiology, 37(2), 204-215. 
7. Bradley, M. M., Codispoti, M., Cuthbert, B. N., \& Lang, P. J. (2001). Emotion and motivation: I. Defensive and appetitive reactions in picture processing. Emotion, 1, 276-298.

8. Brown, P. (2013). Arguing for Media Psychology as a distinct field. En K. Dill (Ed.), The Oxford Handbook of Media Psychology (pp. 474-495). New York: Oxford University Press.

9. Bushman, B. J., \& Anderson, C. A. (2001). Media violence and the American public: Scientific facts versus media misinformation. American Psychologist, 56(6-7), 477.

10. Cantril, A. H, \& Allport, G.W. (1935). The Psychology of Radio. New York: Harper \& Brothers.

11. Cantril, H., Gaudet, H., \& Herzog, H. (1940) The invasion from Mars. Princeton, NJ: Princeton University Press.

12. Comstock, G., \& Scharrer, E. (2010). Media and the American child. San Diego: Academic Press.

13. Craig, R. T. (1999). Communication Theory as a Field. Communication Theory, 9(2), 119-161.

14. Craig, R. T., \& Muller, H. L. (2007). Theorizing communication: readings across traditions. London: SAGE.

15. Crick, N. R., \& Dodge, K. A. (1994). A review and reformulation of social information-processing mechanisms in children's social adjustment. Psychological bulletin, 115(1), 74.

16. Demers, D. (2000). Communication Theory in the $21^{\text {st }}$ Century: Differentiation and convergence. Mass Communication and Society, 3(1), 1-2.

17. Dicken-Garcia, H., \& Viswanath, K. (2002). International Communication History. A special issue of mass communication and Society. London: Lawrence Eribaum Associates.

18. Gerbner, G., \& Gross, L. (1976). Living with television: The violenceprofile. Journal of Communication, $26,76$.

19. Giles, D. (2003). Media psychology. USA: Routledge.

20. Graham, F. K., \& Clifton, R. K. (1966). Heart-rate change as a component of the orienting response. Psychological bulletin, 65(5), 305.

21. Horkheimer, M. (1937). Teoría tradicional y teoría crítica. Barcelona: Paidós UAB, 2009.

22. Knobloch-Westerwick, S. (2014). Choice and preference in media use: Advances in selective exposure theory and research. USA: Routledge, 2014.

23. Lang, A. (1995). What can the heart tell us about thinking? In A. Lang (Ed.), Measuring psychological responses to media (pp. 99-112). Hillsdale, NJ: Lawrence Erlbaum.

24. Lang, A. (2006). Using the limited capacity model of motivated mediated message processing to design effective cancer communication messages. Journal of Communication, 56(s1), S57-S80.

25. Lang, A. (2009). The limited capacity model of motivated mediated message processing. In R. Nabi \& M.B. Oliver (Eds), The SAGE handbook of mass media effects (pp. 193-204). USA: Sage publication.

26. Lang, A. (2013). Discipline in crisis? The shifting paradigm of Mass Communication Research. Communication Theory, 23, 10-24.

27. Lang, A. (2014). Dynamic human-centered communication systems theory. The Information Society. An International Journal, 30, 1.

28. Lang, A., Newhagen, J., \& Reeves, B. (1996). Negative video as structure: Emotion, attention, capacity, and memory. Journal of Broadcasting and Electronic Media, 40, 460-477. 
29. Lang, A., Potter, R.F., \& Bolls, P. (2009). Where psychophysiology meets the media: Taking the effects out of mass communication research. In J. Bryant and M.B. Oliver (Eds.). Media Effects: Advances in Theory and Research (pp. 185-206). Hillsdale, NJ: Lawrence Erlbaum.

30. Lang, A., \& Yegiyan, N. S. (2009). Motivated message processing: How media elicit motivation which influences how media are processed. Biological dimensions of communication, 135-159.

31. Lang, P. J. (1979). A bio-informational theory of emotional imaginery. Psychophyisiology, 16, 495-512.

32. Leshner, G., Bolls, P., \& Thomas, E. (2009). Scare' Em or Disgust 'Em: The Effects of Graphic Health Promotion Messages. Health Communication, 24, 447-458.

33. Martínez, P. (2012). The consumer mind. Brain perception and the implications for marketers. London: MillwardBrown.

34. Mas, Ll., \& Rodero, E. (2015). If communication were a science. Communication as a discipline and as a field. Moscow: National Research University, Higher School of Economics.

35. McQuail, D. (2013). Reflections on paradigm change in communication theory and research. International of Journal of Communication, 7, 216-229.

36. Miller, A. (2003). The cognitive revolution: a historical perspective. Trends in Cognitive Sciences, 7(3), 141-144.

37. Neuman, W. R., \& Guggenheim, L. (2011). The evolution of media effects theory: A Six-Stage model of cumulative research. Communication Theory, 21, 169-196. doi:10.1111/j.1468-2885.2011.01381.x

38. Parsons, T. (1978). Action theory and the human condition. Free Press: New York.

39. Peters, J. D. (1999). Speaking into the air. A History of the idea of Communication. Chicago: University of Chicago press.

40. Potter, R. F., \& Bolls, P. D. (2012). Psychophysiological Measurement and Meaning: Cognitive and Emotional Processing of Media. Routledge Communication series.

41. Potter, W. J. (2013). A general framework for Media Psychology scholarship. In Karen Dill (Ed.), The Oxford Handbook of Media Psychology (pp. 474-495). New York: Oxford University Press.

42. Putnam, L. L. (2001). Shifting voices, oppositional discourse, and new visions for communication studies. Journal of Communication, 51, 38-51.

43. Rodero, E., \& Potter, R. (2015). Melodic variations to stimulate your attention. Communication across the life span. $65^{\text {th }}$ ICA Annual Conference, ICA, Puerto Rico, USA.

44. Shome, R. (2006). Interdisciplinary research and globalization. The Communication Review, 9 (1), 1-36.

45. Vila, J., \& Guerra, P. (2009). Una introducción a la psicofisiología clínica. Madrid: Ediciones Pirámide.

46. Weyers, P., Mühlberger, A., Hefele, C., \& Pauli, P. (2006). Electromyographic responses to static and dynamic avatar emotional facial expressions. Psychophysiology, 43(5), 450-453. 